\title{
actualización
}

\section{Uso de la marihuana medicinal en enfermedades reumáticas}

\section{Darío Scublinsky}

Departamento de Farmacología, Facultad de Medicina, UBA.

Palabras clave:

cannabis, enfermedades reumáticas.

Key words:

cannabis, rheumatic diseases.

Correspondencia

E-mail: darioscublinsky@yahoo.com.ar

\section{R E S U M E N}

La marihuana medicinal ha sido propuesta en los últimos años como una solución a algunos problemas médicos refractarios a otros tratamientos. En este trabajo se realiza una breve revisión desde la historia moderna de los cannabinoides y la farmacología del THC, hasta la evidencia actual de la utilidad de estas sustancias en las enfermedades reumáticas.

\section{A B S T R A C T}

Medical marijuana has been proposed in recent years as a solution to some medical problems refractory to other treatments. In this paper a brief review is made from the modern history of cannabinoids and the pharmacology of THC up to the current evidence of the usefulness of these substances in rheumatic diseases. 


\section{Breve historia del descubrimiento moderno de los cannabinoides}

La marihuana y derivados han sido utilizados como agentes terapéuticos en la medicina oriental, con la evidencia más antigua en la práctica china antigua que data del 2700 a.C. Sin embargo, tan sólo hace 60 años comenzó su camino en la ciencia moderna. Un búlgaro radicado en Jersusalén, el profesor Raphael Mechoulam, comenzó a estudiar los componentes activos de la planta de la marihuana. Al poco tiempo describió una serie de moléculas con tres anillos, cannabinoides, y a una ellas en particular le atribuyó las propiedades psicotrópicas de la marihuana: el delta-9-tetrahidrocanabinol (THC). A continuación descubrió otros cannabinoides activos como el cannabinol (CBN) y el cannabidiol (CBD), pero sin la carga de tener las acciones psicotrópicas del primero. El paso siguiente en la investigación fue identificar si se trataban de sustancias de acción inespecífica como es el alcohol. Pero en ese camino se descubrieron los receptores cannabinoides, lo que dio por certero que el mecanismo de acción era específico. Finalmente, Mechoulam desafió a la Fisiología preguntándose por qué existirían receptores cannabinoides en el organismo. La respuesta no tardó en llegar cuando descubrió uno de sus ligandos endógenos: la anandamida, que dio lugar a la descripción de un nuevo sistema de neurotransmisión llamado "endocannabinoide".

\section{La ciencia y la marihuana medicinal}

La farmacología moderna, a través de sus 80 años de evolución, ha intentado dar respuesta a distintas situaciones fisiopatológicas basada principalmente en la comprensión de los mecanismos de las enfermedades. El descubrimiento de los "receptores moleculares", las "vías de señalización intracelular" y la confirmación de una farmacodinamia específica, han llevado a una tormenta de líneas de investigación y a la explosión de la farmacología molecular y los medicamentos biotecnológicos.

También las formas de experimentación en farmacología han pasado por distintas etapas en las cuales se podría resaltar que, de mediados del siglo XX hacia adelante, a partir de los juicios de Nuremberg y la Declaración de Helsinki, la investigación ha logrado congeniar con la sociedad los principios de interés mutuo, el respeto por el paciente (sujeto de investigación) y de ética aplicada. La farmacovigilancia moderna tuvo un nacimiento más tardío, hacia los años 1960, a partir de la talidomida. Y hoy nadie duda de la necesidad de las 4 fases de la farmacología clínica para la evaluación y aprobación de nuevos medicamentos originales, para maximizar la posibilidad de contar con fármacos más eficaces y seguros.

Pero la realidad de nuestros días nos enfrenta con el hecho de que a pesar de todos los avances médicos, algunos pacientes no pueden encontrar respuestas en los tratamientos que les ofrecemos. En los últimos años se han dado a conocer algunos casos de enfermos que, sin respuesta a la medicina tradicional, respondían a la marihuana o sus derivados más allá de que no existiese una explicación precisa de porqué "funcionaba" en esos pacientes.

Con la promulgación de la Ley del Cannabis medicinal en Argentina y su reglamentación reciente, este tema comienza a ser abordado por todas las especialidades donde su aplicación potencial o efectiva pasa a ser una realidad. La Reumatología es una de estas especialidades.

\section{Apuntes sobre la farmacología del THC}

Volviendo al delta-9-tetrahidrocanabinol del profesor Mechoulam ${ }^{1}$, la cantidad de THC que se absorbe y la velocidad de la absorción depende de la vía de administración. Por inhalación de humo (en vaporizadores, cigarrillos o pipas de agua), la absorción es rápida y la cantidad absorbida depende de la forma de fumar; puede variar entre un $10 \%$ y un $50 \%$ según la profundidad de las inspiraciones y retención de humo en los pulmones. El CBD y el CBN tienen una biodisponibilidad por vía inhalada del $40 \%$. Los efectos se inician en pocos segundos y son completos antes de media hora². Por vía oral, la absorción del THC es lenta, irregular y variable entre los individuos.

La concentración plasmática de THC aumenta rápidamente después de la administración de cannabis por vía inhalada y también desaparece rápidamente del plasma a las 3-4 $\mathrm{h}^{3}$. Hay poca relación entre las concentraciones plasmáticas de THC y sus efectos neuropsicológicos, debido al modelo farmacocinético multicompartimental ${ }^{4}$.

Cuando llega a la circulación sistémica, el THC se distribuye rápidamente por el organismo, primero a los tejidos más irrigados (cerebro, riñón, estómago, pulmones, hígado, corazón, bazo, etc.) y posteriormente se acumula en el tejido adiposo. El THC acumulado en el tejido adiposo se va liberando lentamente a la sangre, de forma que su eliminación completa puede necesitar hasta 30 días. Este patrón de eliminación explicaría que su síndrome de abstinencia no sea tan pronunciado como con otros psicotrópicos ${ }^{5}$.

La mayoría de los estudios sugieren que la vida media del THC es de $56 \mathrm{~h}$ en consumidores ocasionales y de $28 \mathrm{~h}$ en consumidores crónicos ${ }^{6}$. No obstante, en un estudio se registró una vida media en fumadores crónicos, de 3 a 5 días ${ }^{3}$. Lo que queda claro es la existencia de diferencias farmacocinéticas entre el uso ocasional y crónico de cannabis ${ }^{7}$. El THC casi no se detecta en orina y aparece en líquidos biológicos como el sudor, la saliva y el cabello. Los cannabinoides atraviesan la placenta y se excretan por la leche materna durante la lactancia ${ }^{8}$.

\section{Receptores cannabinoides}

En los años ochenta se empezó a trabajar experimentalmente con derivados cannabinoides marcados con isotopos radioactivos, de forma que se pudieron establecer mapas de sus receptores. En 1990 se describió por primera vez la estructura molecular del receptor $\mathrm{CB}^{9}$, que se encuentra principalmente en el sistema nervioso central (SNC), en regiones implicadas en funciones cognitivas, memoria, ansiedad, dolor, percepción visceral, coordinación motora y funciones endocrinas 3,10 .

En 1993 se describió la estructura del receptor CB2 ${ }^{11}$, el cual se encuentra sobre todo en el bazo y las células del sistema inmune, y no se encuentra en el tejido neuronal del SNC. No obstante, se

Figura 1. THC, cannabinoides sintéticos y endocannabinoides.
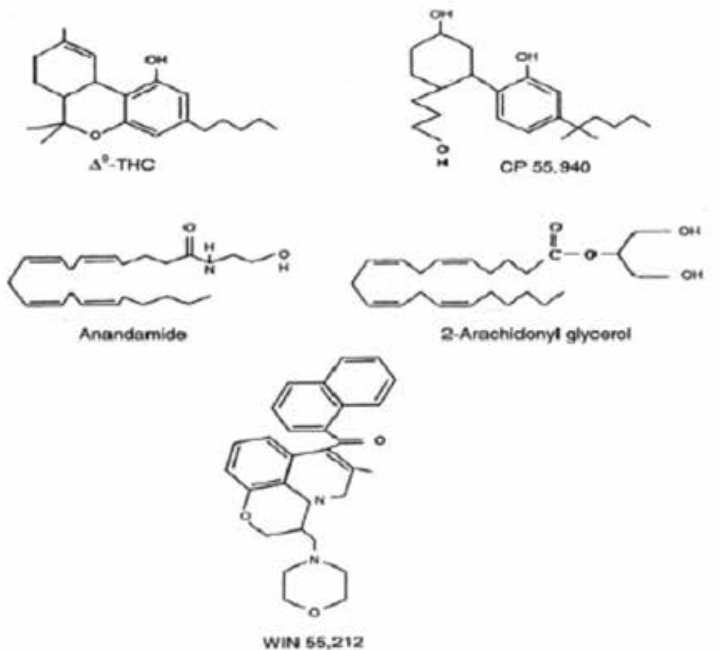
han aislado receptores CB2 en la microglia de rata $^{12}$. Se ha observado que tendría un papel en el efecto inmunomodulador de los cannabinoides.

No se puede descartar la existencia de otros tipos de receptores cannabinoides dado que no todos los efectos de los cannabinoides se pueden explicar por la unión a los receptores CB1 y CB2 ${ }^{13}$.

\section{Cannabinoides endógenos}

Se han identificado tres familias de lípidos endógenos que se unen con más o menos afinidad a los receptores CB1 y CB2 y producen los mismos efectos que el THC en modelos de experimentación animal (antinocicepción, inmovilidad, reducción de la actividad espontánea e hipotermia). Estas sustancias son los cannabinoides endógenos o endocannabinoides:

a) La anandamida es un derivado del ácido araquidónico y es la etanolamida de ácidos grasos poliinsaturados más conocida ${ }^{14}$.

b) El 2-araquidonil-glicerol (2-ARA-G) es, hoy por hoy, el único endocannabinoide del grupo de los esteres del ácido araquidónico ${ }^{15}$.

c) El éter de 2-araquidonil-gliceril o noladina es un tercer tipo de endocannabinoide que ha sido identificado recientemente ${ }^{16}$.

Los endocannabinoides son neurotransmisores, pero no se acumulan en vesículas sinápticas. En el sistema nervioso se comportan mayoritariamente como neuromoduladores inhibiendo la propagación del impulso nervioso, la transmisión sináptica y la liberación de neurotransmisores. Se han relacionado con la dopamina en la modulación de la actividad motora y la secreción de hormonas adenohipofisarias; con la adrenalina, la serotonina, el glutamato y el GABA en mecanismos de aprendizaje y memoria, $\mathrm{y}$ con los péptidos opioides en el control del dolor y los mecanismos de recompensa ${ }^{3}$.

\section{Efectos farmacológicos del cannabis y los cannabinoides}

Los principales efectos de los cannabinoides en el ser humano son los siguientes:

\section{Efectos neuropsiquiátricos}

Los términos con los que se describen los efectos del consumo recreativo de cannabis sobre el sistema nervioso son ligera euforia, relajación, aumento de la sociabilidad, y potenciación de las percepciones sensoriales y de la creatividad. Otros efectos habituales, no tan deseados o buscados con su consumo son alteración de la percepción del paso del tiempo, alucinaciones, psicosis, despersonalización, fragmentación del pensamiento y ansiedad. La mitad de los usuarios habituales han tenido como mínimo un episodio de ansiedad consecuente a su consumo, generalmente a dosis altas ${ }^{6}$. La vía de administración (oral, inhalada o intravenosa) no modifica la naturaleza de estos efectos, pero sí la rapidez de su aparición, su intensidad y su duración, porque la velocidad de absorción y la biodisponibilidad dependen de la vía de administración ${ }^{17}$.

\section{Efecto sobre la memoria}

La marihuana altera la memoria a corto plazo $^{18}$. Este efecto se ha relacionado con la concentración elevada de receptores cannabinoides CB1 en el hipocampo. En estudios en animales se ha observado que los cannabinoides disminuyen la actividad neuronal del hipocampo. Las ratas presentan tolerancia a estos efectos ${ }^{19}$. También se ha observado que estos efectos son reversibles ${ }^{20}$.

En estudios experimentales se ha observado que ratones transgénicos sin receptores cerebrales CB1 (ratones Knock out) presentan un aumento de la memoria ${ }^{21} \mathrm{y}$ tienen dificultad para olvidar la sensación de miedo asociada a un estímulo aversivo en comparación con los ratones controles ${ }^{22}$.

\section{Efecto sobre la actividad motora}

La marihuana afecta a la actividad motora. Los cannabinoides son mayoritariamente inhibidores de la transmisión y de la coordinación motora a través de su acción en los ganglios basales y el cerebelo, donde hay una densidad elevada de receptores CB1 ${ }^{23}$. Este efecto depende del tipo de trabajo y la experiencia previa con la marihuana. En general, se afectan trabajos motores que requieren atención como escribir a mano, conducir o el uso de maquinaria ${ }^{24-27}$.

\section{Efecto antiemético}

El mecanismo antiemético exacto del cannabis y sus derivados se desconoce. Se han identificado receptores CB1 en el núcleo del tracto solitario en el SNC ${ }^{28}$. Este núcleo, situado en el tronco cerebral, se ha implicado en la regulación de la emesis ${ }^{29}$. Estudios recientes han mostrado que los cannabinoides inhiben el vómito. Estos estudios sugieren que varios cannabinoides tendrían efecto antinauseoso y antiemético, y que estos efectos estarían mediados por mecanismos diferentes y tendrían un efecto antiemético aditivo con los inhibidores de la serotonina ${ }^{30-33}$.

\section{Evidencia del uso de cannabis en enfermedades reumáticas}

En el análisis de la evidencia del uso clínico del cannabis, sus preparados y derivados, surgen de la bibliografía indexada varios trabajos con distintos niveles de evidencia, la mayoría de ellos con niveles muy bajos de la misma, dados por estudios abiertos, casos clínicos, diseños no controlados y no ciegos. Por otro lado, se encuentra el problema de las distintas preparaciones, distintas dosis y vías de administración. No existen estudios clínicos con diseños aceptables del uso de aceite de cannabis para alguna enfermedad reumática. Los trabajos actuales con mayor evidencia disponible son dos revisiones sistemáticas de grupos independientes y una revisión de Cochrane, que se describen a continuación.

Uno es el trabajo de Fitzcharles MA y cols..$^{34}$, donde se incluyeron dos ECA de 2 y 4 semanas de duración respectivamente con nabilona, incluidos 71 pacientes con FMS; un ensayo de 4 semanas con nabilona, incluidos 30 pacientes con dolor espinal, y un estudio de 5 semanas con tetrahidrocannbinol/cannabidiol, incluidos 58 pacientes con AR. Un criterio de inclusión fue el dolor refractario al tratamiento convencional en tres estudios. No se encontraron ECA en pacientes con OA. El riesgo de sesgo fue alto para los tres estudios. Los hallazgos de una superioridad de los cannabinoides sobre los controles (placebo, amitriptilina) no fueron consistentes. Los cannabinoides fueron generalmente bien tolerados a pesar de algunos efectos secundarios conocidos para estas drogas. Por lo que concluyen que, actualmente, no hay pruebas suficientes para recomendar cualquier preparación de cannabinoides para el tratamiento de los síntomas en pacientes con dolor crónico asociado con enfermedades reumáticas.

En el otro trabajo ${ }^{35}$ se realizaron búsquedas en varias bases de datos, incluidas Medline, Embase y CENTRAL. Se incluyeron ensayos controlados aleatorios con resultados de dolor, sueño, calidad de vida, tolerabilidad (abandonos debidos a eventos adversos) y seguridad (eventos adversos graves), con comparación de cannabinoides con cualquier tipo de control. La calidad de la metodología del estudio se evaluó con la herramienta Cochrane de riesgo de sesgo. Los resultados arrojados fueron que, en 4 estudios a corto plazo que incluyeron 203 pacientes ( 58 con artritis reumatoidea, 71 con fibromialgia y 74 con osteoartritis [OA]), los cannabinoides tuvieron un efecto estadísticamente significativo sobre el dolor en 2, dormir en 2 y mejorar la calidad de vida en 1 , con el estudio de OA prematuramente terminado debido a futili- 
dad. El riesgo de sesgo fue alto para los 3 estudios completados. Se informaron mareos, problemas cognitivos y somnolencia, así como náuseas, en casi la mitad de los pacientes. No se informaron eventos adversos graves para los cannabinoides durante la duración del estudio. No se identificaron estudios de cannabis herbal.

En definitiva, el estudio concluye que: Los tamaños de muestra extremadamente pequeños, la corta duración del estudio, la heterogeneidad de las condiciones reumáticas, los productos, y la ausencia de estudios de cannabis herbal permiten conclusiones solo limitadas para los efectos de los cannabinoides en condiciones reumáticas. El alivio del dolor y el efecto sobre el sueño pueden tener algún beneficio terapéutico potencial, pero con frecuentes eventos adversos de leves a moderados.

Por último, en la revisión de Cochrane del uso de cannabis en fibromialgia ${ }^{36}$, se incluyeron dos estudios con 72 participantes. En general, los dos estudios tenían un riesgo moderado de sesgo. La evidencia se derivó de los datos de la media del grupo y del análisis completo (evidencia de muy baja calidad en general). Se calificó la calidad de todos los resultados según GRADE como muy baja, debido a la imprecisión y el posible sesgo de notificación. Los resultados primarios de la revisión fueron un alivio del dolor informado por el participante del $50 \%$ o más, impresión global de cambio del paciente (PGIC) o mucho mejor, retiro debido a eventos adversos (tolerabilidad) y eventos adversos graves (seguridad). La nabilona se comparó con placebo y con amitriptilina en un estudio cada uno. Los tamaños del estudio fueron de 32 y 40 participantes. Un estudio utilizó un diseño cruzado y uno usó un diseño de grupos paralelos; la duración del estudio fue de cuatro o seis semanas. Ambos estudios usaron nabilona, un cannabinoide sintético, con una dosis para la hora de acostarse de 1 $\mathrm{mg} /$ día. Ningún estudio informó la proporción de participantes que experimentaron al menos un $30 \%$ o $50 \%$ de alivio del dolor, o que mejoraron mucho. Ningún estudio proporcionó evidencia de primer o segundo nivel (calidad alta a moderada) para un resultado de eficacia, tolerabilidad y seguridad. La evidencia de tercer nivel (de muy baja calidad) indicó una mayor reducción del dolor y las limitaciones de la CVRS en comparación con el placebo en un estudio. No hubo diferencias significativas con respecto al placebo observado para la fatiga y la depresión (evidencia de muy baja calidad). La evidencia de tercer nivel indicó mejores efectos de la nabilona en el sueño que la amitriptilina (evidencia de muy baja calidad). No hubo diferencias significativas entre los dos medicamentos señalados para el dolor, el estado de ánimo y la CVRS (evidencia de muy baja calidad). Más participantes abandonaron debido a eventos adversos en los grupos de nabilona (4/52 participantes) que en los grupos control (1/20 en el grupo placebo y 0/32 en el grupo amitriptilina). Los eventos adversos más frecuentes fueron mareos, náuseas, sequedad de boca y somnolencia (seis participantes con nabilona). Ninguno de los estudios informó eventos adversos graves durante el período de ambos estudios. Los autores planearon crear una tabla GRADE de "Resumen de hallazgos", pero debido a la escasez de datos no pudieron hacerlo. No encontraron ningún estudio relevante con cannabis herbal, cannabinoides de origen vegetal o cannabinoides sintéticos distintos de la nabilona en la fibromialgia.

Por lo tanto, al momento se puede concluir que no hay pruebas suficientes para recomendar cannabinoides en el tratamiento de enfermedades reumáticas. Si bien existen casos que llegan de pacientes que se encuentran consumiendo cannabinoides y manifiestan respuestas favorables, los pacientes que no presentan ese tipo de respuestas suelen callar sobre la experiencia, por lo cual existe un marcado sesgo de comunicación, más allá de las distintas variables comentadas y cuantiosas imprecisiones en la medición de las respuestas fuera de estudios clínicos con un diseño ético y metodológico adecuados.

Pero lo más significativo en el campo de la aplicación médica del cannabis en la Argentina es que no resultan claros los métodos de manufactura de los cannabinoides en el país, tampoco los controles de calidad y de la distribución del producto, las dosis, las vías de administración, así como tampoco se cuenta con estudios de farmacocinética, farmacodinamia, eficacia ni seguridad. Por tales motivos, en las condiciones actuales, no resulta adecuada la utilización de estas sustancias en las enfermedades reumáticas.

\section{BIBLIOGRAFÍA}

1. Mechoulam R, Gaoni Y. A total synthesis of dl-delta1-tetrahydrocannabinol, the active constituent of hashish. J Am Chem Soc. 1965 Jul 20;87:3273-5.

2. Lindgren JE, Ohlsson A, Agurell S, Hollister L, Gillespie H. Clinical effects and plasma levels of delta 9-tetrahydrocannabinol (delta 9-THC) in heavy and light users of cannabis. Psychopharmacology 1981; 74:208-12.

3. Ramos Atance JA, Fernández Ruiz J. Cannabinoides: propiedades químicas y aspectos metabólicos. Adicciones 2000; 12 (supl 2):41-56.

4. Grotenhermen F. Pharmacokinetics and pharmacodynamics of cannabinoids. Clin Pharmacokinet 2003; 42:327-60.

5. Williamson EM, Evans F. Cannabinoids in clinical practice. Drugs 2000; 60:1303-14.

6. Ashton $\mathrm{CH}$. Adverse effects of cannabis and cannabinoids. Br J Anaesth 1999; 83:637-49.

7. Martín BR, Dewey WL, Harris LS, Belckner JS. Delta-9tetrahydrocannabinol tissue and subcellular distribution in the central nervous system and tissue distribution in peripheral organs of tolerant and nontolerant dogs. J Pharmacol Exp Ther 1976; 196:128-44.

8. Fernandez-Ruiz JJ, Rodríguez F, Navarro M, Ramos JA. Maternal cannabinoid exposure and brain development: changes in the ontogeny of dopamine neurons: Bartke A, Murphy LL (eds). Marihuana/cannabinoids: neurobiology and neurophysiology. Biochemistry and physiology of substance abuse: CRC Press 1992; 4:119-64.

9. Matsuda LA, Lolait SJ, Brownstein MJ, Young AC, Bonner TI. Structure of a cannabinoid receptor and functional expression of the cloned cDNA. Nature 1990; 346:561-64.

10. Pertwee RG. Pharmacology of cannabinoid CB1 and CB2 receptors. Pharmacol Ther 1997; 74:129-80.

11. Munro S, Thomas KL, Abu-Shaar M. Molecular characterisation of peripheral receptor for cannabinoids. Nature 1993; 365:61-65.

12. Pertwee RG. Cannabinoid receptors and pain. Progress in Neurobiology 2001; 63:569-611.

13. Pertwee RG. Pharmacology of cannabinoid receptor ligands. Curr Med Chem 1999; 6:635-64.

14. Mechoulam R, Ben Shabat S, Hanus L, Fride E, Vogel Z, Bayewitch $M$ et al. Endogenous cannabinoid ligandschemical and biological studies. J Lipid Mediat Cell Signal 1996; 14:45-49.

15. Mechoulam R, Ben-Shabat S, Hanus L, Ligumsky M, Kaminski NE, Schatz AR et al. Identification of an endogenous 2-mono-glyceride, present in canine gut, that binds to cannabinoid receptors. Biochem Pharmacol 1995; 50:83-90.

16. Hanus L, Abu-Lafi S, Fride E, Breuer A, Vogel Z, Shalev DE et al. 2-arachidonil gliceril eter, an endogenous agonist of the cannabinoid CB1 receptor. Proc Natl Acad Sci USA 2000; 98:3662-35.

17. Perz-Reyes M. The psychologic and physiologic effects of active cannabinoids. A: Nahas G, Sutin KM, Harvey DJ, Agurell S (eds), Marihuana and medicine. Totowa, NJ: Humana Press, 1999:245-52.

18. Dewey WL. Cannabinoid pharmacology. Pharmacol Rev 1986; 38:151-78.

19. Kirby MT, Hampson RE, Deadwyler SA. Cannabinoids selectively decrease paired-pulse facilitation of 
perforant path synaptic potentials in the dentate gyrus in vitro. Brain Res 1995; 688:114-20.

20. Nakamura EM, Da Silva EA, Concilio GV, Wilkinson DA, Masur J. Reversible effects of acute and long-term administration of delta9- tetrahydrocannabinol on memory in the rat. Drug Alcohol Depend 1991; 28:167-75.

21. Reibaud $M$, Obinu MC, Ledent $C$, Parmentier $M$, Böhme GA, Imperato A. Enhancement of memory in cannabinoid CB1 receptor knockout mice. Eur J Pharmacol 1999; 379:R1-R2.

22. Marsiciano G. The endogenous cannabinoid system controls extinction of aversive memories. Nature 2002; 418:530-34.

23. Pertwee RG, Wickens AP. Enhancement by chlordiazepoxide of catalepsy induced in rats by intravenous or intrapallidal injections of enantiomeric cannabinoids. Neuropharmacology 1991; 30:237-44.

24. Ramaekers JG, Berghaus $G$, van Laar $M$, Drummer $\mathrm{OH}$. Dose related risk of motor vehicle crashes after cannabis use. Drug Alcohol Depend 2004; 73:109-19.

25. Drummer $\mathrm{OH}$, Gerostamoulos J, Batzirs H, Chu M, Caplehorn J, Robertsn MD, Swann P. The involvement of drugs in drivers of motor vehicles killed in Australian road traffic crashes. Accid Anal Prev 2004; 36:239-48.

26. Bates MN, Blakey TA. Role of cannabis in motor vehicle crashes. Epidemiol Rev 1999; 21:222-32.

27. Longo MC, Hunter CE, Lokan RJ, White JM, White MA. The prevalence of alcohol, cannabinoids, benzodiazepines and stimulants amongst injured drivers and their role in driver culpability. Part II: the relation between drug prevalence and drug concentration, and driver culpability. Accid Anal Prev 2000; 32:623-32.

28. Van Sickle MD, Oland LD, Ho W, Hillard CJ, Mackie $\mathrm{K}$, Davison JS et al. Cannabinoids inhibit emesis through CB1 receptors in the brainstem of the ferret. Gastroenterology 2001; 121:767-74.

29. Fan P. Cannabinoid agonists inhibit the activation of 5-HT3 receptors in rat nodose ganglion neurons. J Neurophysiol 1995; 73:907-10.

30. Parker AL, Mechoulam R, Schlievert C. Cannabidiol, a non-psychoactive component of cannabis and its synthetic dimethylheptyl homolog suppress nausea in an experimental model with rats. Neuroreport 2002; 13:567-70.
31. Parker LA, Mechoulam R, Schlievert C, Abbott L, Fudge ML, Burton P. Effects of cannabinoids on lithiuminduced conditioned rejection reactions in a rat model of nausea. Psychopharmacology 2003; 166:156-62.

32. Parker LA, Kwiatkowska M, Burton P, Mechoulam R. Effect of cannabinoids on lithium-induced vomiting in the Suncus murinus (house musk shrew). Psychopharmacology 2004;171:156-61.

33. Kwiatkowska M, Parker LA, Burton P, Mechoulam R. A comparative analysis of the potential of cannabinoids and ondansetron to suppress cisplatin-induced emesis in the Suncus murinus. Psychopharmacolgy 2004.

34. Fitzcharles MA, Baerwald C, Ablin J, Häuser W. Efficacy, tolerability and safety of cannabinoids in chronic pain associated with rheumatic diseases (fibromyalgia syndrome, back pain, osteoarthritis, rheumatoid arthritis): A systematic review of randomized controlled trials. Schmerz. 2016 Feb;30(1):47-61. doi: 10.1007/ s00482-015-0084-3.

35. Fitzcharles MA, Ste-Marie PA, Häuser W, Clauw DJ, Jamal S, Karsh J, Landry T, Leclercq S, Mcdougall JJ, Shir Y, Shojania K, Walsh Z. Efficacy, Tolerability, and Safety of Cannabinoid Treatments in the Rheumatic Diseases: A Systematic Review of Randomized Controlled Trials. Arthritis Care Res (Hoboken). 2016 May;68(5):681-8. doi: 10.1002/acr.22727.

36. Walitt B, Klose P, Fitzcharles MA, Phillips T, Häuser W. Cannabinoids for fibromyalgia. Cochrane Database Syst Rev. 2016 Jul 18;7:CD011694. doi:10.1002/14651858. CD011694.pub2. 